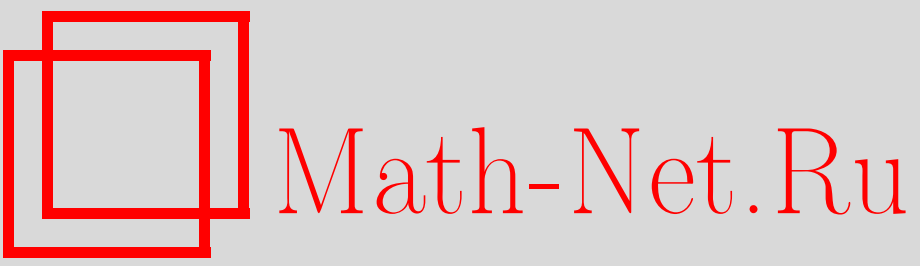

В. И. Фернандес, Ч. М. Наон, Процессы рассеяния назад и UMKLAPPрассеяния для одномерных бесспиновых фермионов в формулировке интеграла по путям, ТМФ, 1999, том 118, номер 3, 488-496

DOI: https://doi.org/10.4213/tmf722

Использование Общероссийского математического портала Math-Net.Ru подразумевает, что вы прочитали и согласны с пользовательским соглашением

http: //www.mathnet.ru/rus/agreement

Параметры загрузки:

IP: 54.237 .206 .68

26 апреля 2023 г., $17: 29: 36$ 


\section{ПРОЦЕССЫ РАССЕЯНИЯ НАЗАД \\ И UMKLAPР-PAССЕЯНИЯ ДЛЯ ОДНОМЕРНЫХ БЕССПИНОВЫХ ФЕРМИОНОВ В ФОРМУЛИРОВКЕ ИНТЕГРАЛА ПО ПУТЯМ}

Рассмотрена $(1+1)$-мерная фермионная квантовая теория поля с нелокальным взаимодействием между токами. Эта модель описывает ансамбль бесспиновых фермионов, взаимодействующих посредством процессов рассеяния назад и вперед и процесса рассеяния UMKLAPP. Функционал перехода вакуум-вакуум удается выразить через нетривиальный фермионный детерминант. Используя методы функционального интегрирования, мы нашли бозонное представление для этого детерминанта и соответствующее эффективное действие, зависящее от трех скалярных полей, два из которых отвечают физическим коллективным возбуждениям, а третье - вспомогательное поле, по которому можно проинтегрировать с помощью техники приближений.

\section{1. ВВЕДЕНИЕ}

В настоящее время интерес к маломерным полевым теориям переживает очередной всплеск, связанный, в частности, с исследованием одномерного фермионного газа, которое имеет прямое отношение к промышленному производству так называемых квантовых проводов [1]. Одно из наиболее интересных свойств таких систем - это возможность поведения, отличного от поведения типа жидкости Ферми. Это явление было систематически исследовано Халдейном в работе [2], который предложил термин “жидкость Люттингера", чтобы выделить это новое физическое состояние, в котором поверхность Ферми исчезает и спектр содержит только коллективные возбуждения. Вероятно, наиболее простая модель, проявляющая такие свойства, - это модель Томонаги-Люттингера [3], являюшаяся многочастичной системой право- и леводвигаюшихся частиц, взаимодействующих через их плотности зарядов. В работе [4] был предложен альтернативный теоретико-полевой подход к этой проблеме. В этом подходе вводится нелокальное нековариантное обобщение модели Тирринга $[5,6]$, в которой фермионные плотности и токи спариваются с помошью билокальных потенциалов, зависяших от

\footnotetext{
*Depto. de Física. Universidad Nacional de La Plata. CC 67, 1900 La Plata, Argentina.

${ }^{\dagger}$ Consejo Nacional de Investigaciones Científicas y Técnicas, Argentina. E-mail: naon@venus.fisica.unlp.edu.ar
} 
расстояния. Эта нелокальная модель Тирринга (НМТ) содержит как частньй случай модель Томонаги-Люттингера. Хотя НМТ и является мошным методом изучения одномерных многочастичных задач, ее применение к описанию квантовых проводов ограничено, поскольку в теории возмушений составляющие НМТ суть процессы рассеяния вперед (fs), которые дают главный вклад только при малом переданном импульсе. Следовательно, в своем настояшем виде НМТ дает лишь весьма грубое описание процессов установления равновесия и свойств переноса в жидкости Люттингера. В настоящей работе мы рассматриваем улучшенную версию НМТ, в которой учитываются процессы с большим переданным импульсом. Заметим, что наиболее интересные проявления этих процессов типа появления массовых щелей в спектрах нормальных мод характерны для случая теорий с частицами спина $1 / 2$, в данной работе для наглядности мы ограничиваемся бесспиновым случаем. В разделе 2 мы покажем, что преобразования, позволяющие выписать вакуумный функционал проблемы рассеяния вперед через фермионный детерминант, справедливы и в рамках НMT даже с учетом процессов рассеяния назад (bs) [7] и процессов UMKLAPP (us) [8]. Мы находим фермионный детерминант специального вида, который напоминает детерминант, получаемый при исследовании методом функционального интеграла [9] массивных фермионов в размерности $1+1$ [10]. В разделе 3 мы опишем бозонное представление для этого фермионного детерминанта. Это, в свою очередь, позволяет получать полностью бозонизованное эффективное действие для рассматриваемой модели. Наконец, в разделе 4 кратко обсуждаются основные результаты нашего исследования.

\section{2. ФОРМУЛИРОВКА МОДЕЛИ}

Начнем исследование расширенной версии НМТ, включающей вклады от процессов рассеяния вперед, назад и от процессов UMKLAPP. Следуя формулировке задачи, приведенной в работе [4], мы опишем эти взаимодействия через фермионную квантовую теорию поля (КТП) с евклидовым действием

$$
S=S_{0}+S_{\mathrm{fs}}+S_{\mathrm{bs}}+S_{\mathrm{us}}
$$

где

$$
S_{0}=\int d^{2} x \bar{\Psi} i \partial \Psi
$$

- невозмущенное действие, порождающее линеаризованное свободное соотношение дисперсии. Вклады от различных процессов рассеяния имеют вид

$$
S_{\mathrm{fS}}=-\frac{g^{2}}{2} \int d^{2} x d^{2} y\left(\bar{\Psi} \gamma_{\mu} \Psi\right)(x) V_{(\mu)}(x, y)\left(\bar{\Psi} \gamma_{\mu} \Psi\right)(y)
$$

и

$$
S_{\mathrm{bs}}+S_{\mathrm{us}}=-\frac{g^{\prime 2}}{2} \int d^{2} x d^{2} y\left(\bar{\Psi} \Gamma_{\mu} \Psi\right)(x) U_{(\mu)}(x, y)\left(\bar{\Psi} \Gamma_{\mu} \Psi\right)(y),
$$


где $\gamma_{\mu}$ - обычные двумерные матришы Дирака, $\Gamma_{0}=1$ и $\Gamma_{1}=\gamma_{5}$. По повторяюшемуся индексу $\mu$ суммирования не проводится. Потенциалы спариваний $V_{(\mu)}$ и $U_{(\mu)}$ предполагаются зависяшими лишь от расстояния $|x-y|$ и могут быть выражены в терминах сольемовской "g-ологии" [11] в следующем виде:

$$
\begin{gathered}
V_{(0)}(x, y)=\frac{1}{g^{2}}\left(g_{2}+g_{4}\right)(x, y), \\
V_{(1)}(x, y)=\frac{1}{g^{2}}\left(g_{2}-g_{4}\right)(x, y) ; \\
U_{(0)}(x, y)=\frac{1}{g^{\prime 2}}\left(g_{3}+g_{1}\right)(x, y), \\
U_{(1)}(x, y)=\frac{1}{g^{\prime 2}}\left(g_{3}-g_{1}\right)(x, y),
\end{gathered}
$$

где $g$ и $g^{\prime}$ - числовые константы, которые можно положить равными единице. Мы удерживаем их в ответах, для того чтобы было легче сравнивать наши результаты с результатами, полученными в обычной модели Тирринга, которая получается, если положить $g^{\prime}=0$ и $V_{(0)}(x, y)=V_{(1)}(x, y)=\delta^{2}(x-y)$. С другой стороны, в нековариантном пределе $g^{\prime}=0, V_{(1)}(x, y)=0$ появится модель Томонаги-Люттингера [3].

Члены в действии, содержашие $g_{2}$ и $g_{4}$, соответствуют событиям рассеяния вперед, при которых соответствуюший переданный импульс мал. В процессах $g_{2}$ взаимодействуют левые и правые частицы, в то время как в процессах $g_{4}$ все четыре взаимодействующих электрона относятся к одной ветви. Процессы $g_{1}$ и $g_{3}$, напротив, отвечают процессам рассеяния с большими переданными импульсами порядка $2 k_{F}$ (bs) и $4 k_{F}$ (us), coответственно. (Последний вклад значителен только, если зона наполовину заполнена.) Перейдем теперь к рассмотрению статистической суммы. В работе [4] член в действии, отвечаюший вкладу рассеяния вперед, был записан в локальном виде:

$$
S_{\mathrm{fs}}=-\frac{g^{2}}{2} \int d^{2} x J_{\mu} K_{\mu},
$$

где $J_{\mu}$ - обычный фермионный ток, а $K_{\mu}$ - новый ток, задаваемьй формулой

$$
K_{\mu}(x)=\int d^{2} y V_{(\mu)}(x, y) J_{\mu}(y)
$$

После введения функциональной дельта-функции и соответствуюших вспомогательных бозонных полей мы запишем функциональное представление для статистической суммы $Z$ в виде (детали см. в [4])

$$
\begin{aligned}
Z= & N \int \mathcal{D} \bar{\Psi} \mathcal{D} \Psi \mathcal{D} \widetilde{A}_{\mu} \mathcal{D} \widetilde{B}_{\mu} \times \\
& \times \exp \left\{-\int d^{2} x\left[\bar{\Psi} i \partial \Psi+\widetilde{A}_{\mu} \widetilde{B}_{\mu}+\frac{g}{\sqrt{2}}\left(\widetilde{A}_{\mu} J_{\mu}+\widetilde{B}_{\mu} K_{\mu}\right)\right]\right\} .
\end{aligned}
$$


Задавая

$$
\begin{aligned}
\bar{B}_{\mu}(x) & =\int d^{2} y V_{(\mu)}(y, x) \widetilde{B}_{\mu}(y), \\
\widetilde{B}_{\mu}(x) & =\int d^{2} y b_{(\mu)}(y, x) \bar{B}_{\mu}(y),
\end{aligned}
$$

где $b_{(\mu)}(y, x)$ удовлетворяет уравнению

$$
\int d^{2} y b_{(\mu)}(y, x) V_{(\mu)}(z, y)=\delta^{2}(x-z),
$$

и вводя вспомогательные переменные

$$
\begin{aligned}
& A_{\mu}=\frac{1}{\sqrt{2}}\left(\widetilde{A}_{\mu}+\bar{B}_{\mu}\right), \\
& B_{\mu}=\frac{1}{\sqrt{2}}\left(\widetilde{A}_{\mu}-\bar{B}_{\mu}\right),
\end{aligned}
$$

мы получим

$$
Z=N \int \mathcal{D} \bar{\Psi} \mathcal{D} \Psi \mathcal{D} A_{\mu} \mathcal{D} B_{\mu} e^{-S(A, B)-S_{\mathrm{bs}}-S_{\text {us }}} \exp \left\{-\int d^{2} x \bar{\Psi}(i \boldsymbol{\partial}-g \mathbf{A}) \Psi\right\},
$$

где

$$
S(A, B)=\frac{1}{2} \int d^{2} x d^{2} y b_{(\mu)}(x, y)\left[A_{\mu}(x) A_{\mu}(y)-B_{\mu}(x) B_{\mu}(y)\right] .
$$

Якобиан замены $(\widetilde{A}, \widetilde{B}) \rightarrow(A, B)$ не зависит от полей и потому может быть отнесен к константе нормировки $N$. Более того, мы видим, что поле $B$ полностью отщепилось как от поля $A$, так и от фермионного поля. Отсюда интересно восстановить статистическую сумму обычной ковариантной модели Тирринга $\left(b_{(0)}(y, x)=b_{(1)}(x, y)=\delta^{2}(x-y)\right.$ и $\left.g^{\prime}=0\right)$ начиная с интеграла $(2.15)$. Заметим, что $B_{\mu}$ описьвает состояния с отрицательной метрикой, вклады от которых должны быть отфакторизованы и включены в $N$, если только нас интересует физически значимый ответ для $Z$. В функциональном подходе эта процедура идет в параллель с операторным подходом Клейбера [6], в котором использовалось гильбертово пространство с индефинитной метрикой. В дальнейшем мы рассматриваем лишь вклады поля $A$.

Тем самым при выключенных процессах рассеяния назад и UMKLAPP описанная процедура позволяет выразить $Z$ через фермионный детерминант. Покажем теперь, что это возможно также и в случае, если учитываются процессы с большими переданными импульсами. Для этого напишем

$$
S_{\mathrm{bs}}+S_{\mathrm{us}}=-\frac{g^{\prime 2}}{2} \int d^{2} x L_{\mu} M_{\mu}
$$

где $L_{\mu}$ и $M_{\mu}$ - фермионные билинейные комбинации:

$$
\begin{aligned}
L_{\mu}(x) & =\bar{\Psi}(x) \Gamma_{\mu} \Psi(x), \\
M_{\mu}(x) & =\int d^{2} y U_{(\mu)}(x, y) L_{\mu}(y) .
\end{aligned}
$$


Таким образом, мы можем использовать ту же процедуру, что и раньше с $L_{\mu}$ и $M_{\mu}$, играюшими те же роли, что и поля $J_{\mu}$ и $K_{\mu}$, соответственно. После устранения вновь появившихся состояний с отрицательной метрикой и отнесения их статистической суммы в нормировочный множитель мы получим

$$
Z=N \int \mathcal{D} A_{\mu} \mathcal{D} C_{\mu} \operatorname{det}\left(i \boldsymbol{\partial}-g \mathbf{A}-g^{\prime} \Gamma_{\mu} C_{\mu}\right) \exp \{-S[A]-S[C]\},
$$

где

$$
\begin{aligned}
S\left[A_{\mu}\right] & =\frac{1}{2} \int d^{2} x d^{2} y A_{\mu}(x) b_{(\mu)} A_{\mu}(y), \\
S\left[C_{\mu}\right] & =\frac{1}{2} \int d^{2} x d^{2} y C_{\mu}(x) d_{(\mu)} C_{\mu}(y)
\end{aligned}
$$

и

$$
\int d^{2} y d_{(\mu)}(y, x) U_{(\mu)}(z, y)=\delta^{2}(x-z) .
$$

Это наш первый интересный результат: мы представили $Z$ в виде фермионного детерминанта. Однако этот детерминант весьма нетривиален. На самом деле, вклад $g^{\prime}-$ это не только член типа массового (который диагонален в пространстве матриц Дирака), но этот член также зависит от вспомогательного поля $C_{\mu}(x)$. Мы рассматриваем этот детерминант ниже.

\section{3. БОЗОНИЗАЦИЯ ДЕТЕРМИНАНТА}

В настоящем разделе мы делаем киральное преобразование в фермионной мере функционального интегрирования и используем формальное разложение по $g^{\prime}$, для того чтобы найти бозонизованное представление для фермионного детерминанта, найденного в предыдушем разделе. Сделаем следующее преобразование:

$$
\begin{gathered}
\Psi(x)=\exp \left\{g\left[\gamma_{5} \Phi(x)-i \eta(x)\right]\right\} \chi(x), \\
\bar{\Psi}(x)=\bar{\chi}(x) \exp \left\{g\left[\gamma_{5} \Phi(x)+i \eta(x)\right]\right\} ; \\
\mathcal{D} \bar{\Psi} \mathcal{D} \Psi=J_{F}[\Phi, \eta] \mathcal{D} \bar{\chi} \mathcal{D} \chi,
\end{gathered}
$$

где $\Phi$ и $\eta$ - скалярные поля и $J_{F}[\Phi, \eta]$ - якобиан Фуджикавы [12], который отличен от единицы, поскольку (квантовая) мера интегрирования в функциональном интеграле неинвариантна при киральных преобразованиях. Как хорошо известно, после этого преобразования поле $A_{\mu}$ отщепляется от фермионных полей, если представить его в виде

$$
A_{\mu}(x)=\partial_{\mu} \eta(x)+\epsilon_{\mu \nu} \partial_{\nu} \Phi(x),
$$

который также может интерпретироваться как бозонная замена переменных с постоянным, не зависящем от полей якобианом. В результате получим

$$
\operatorname{det}\left(i \boldsymbol{\partial}-g \mathbf{A}-g^{\prime} \Gamma_{\mu} C_{\mu}\right)=J_{F}[\Phi, \eta] \operatorname{det}\left(i \boldsymbol{\partial}-g^{\prime} e^{2 g \gamma_{5} \Phi} \Gamma_{\mu} C_{\mu}\right) .
$$


Чтобы вычислить фермионный якобиан, необходимо фиксировать процедуру регуляризации, что, в свою очередь, привносит дополнительную неопределенность (см., например, [13]). В настоящей работе, мы следуем предписанию работы [13], в которой физический ответ был получен для случая, когда присутствует только рассеяние вперед [4]. В нашем случае находим

$$
J_{F}[\Phi, \eta]=\exp \left\{\frac{g^{2}}{2 \pi} \int d^{2} x \Phi \partial_{\mu} \partial_{\mu} \Phi\right\}
$$

Статистическая сумма имеет при этом вид

$$
Z=N^{\prime} \int \mathcal{D} \Phi \mathcal{D} \eta \mathcal{D} C_{\mu} e^{-\left(S[\Phi, \eta]+S\left[C_{\mu}\right]\right)} J_{F}[\Phi, \eta] \operatorname{det}\left(i \boldsymbol{\partial}-g^{\prime} e^{2 g \gamma_{5} \Phi} \Gamma_{\mu} C_{\mu}\right)
$$

где $S[\Phi, \eta]$ возникает при подстановке $(3.3)$ в функционал $S\left[A_{\mu}\right]$ (см. уравнение $(2.21)$ ).

В полученных формулах фермионный детерминант может быть проанализирован по теории возмущений. В самом деле, рассматривая $g^{\prime}$ как параметр возмушения и используя фермионные поля $\chi$ и $\bar{\chi}$, определенные в (3.1), мы можем записать

$$
Z_{F}=\sum_{n=0}^{\infty} \frac{g^{\prime n}}{n !}\left\langle\prod_{j=1}^{n} \int d^{2} x_{j} \bar{\chi}\left(x_{j}\right) \widehat{C}\left(x_{j}\right) \chi\left(x_{j}\right)\right\rangle_{0},
$$

где мы ввели определения

$$
Z_{F}=\operatorname{det}\left(i \boldsymbol{\partial}-g^{\prime} e^{2 g \gamma_{5} \Phi} \Gamma_{\mu} C_{\mu}\right)
$$

$$
\widehat{C}=\left(\begin{array}{cc}
C_{+} & 0 \\
0 & C_{-}
\end{array}\right)
$$

$$
\begin{aligned}
& C_{+}=\left(C_{0}+C_{1}\right) e^{2 g \Phi(x)}, \\
& C_{-}=\left(C_{0}-C_{1}\right) e^{-2 g \Phi(x)}
\end{aligned}
$$

Рассматривая по отдельности каждый член разложения, мы можем сформулировать правило отбора, сходное с правилами отбора, получающимися при функциональном рассмотрении $(1+1)$-мерной теории массивных фермионов с локальным [9] и нелокальным [14] взаимодействиями. В самом деле, т.к. \langle\rangle$_{0}-$ это вакуумное среднее по отношению к вакууму свободных безмассовых фермионов, то средние, отвечающие в формуле (3.7) членам с $n=2 k+1$, обрашаются в нуль. Таким образом, мы получим

$$
\begin{aligned}
Z_{F}= & \sum_{k=0}^{\infty} \frac{\left(g^{\prime} c \rho\right)^{2 k}}{(k !)^{2}(2 \pi)^{2 k}} \int \prod_{i=1}^{k} d^{2} x_{i} d^{2} y_{i} \prod_{i=1}^{k}\left(C_{0}\left(x_{i}\right)+C_{1}\left(x_{i}\right)\right)\left(C_{0}\left(y_{i}\right)-C_{1}\left(y_{i}\right)\right) \times \\
& \times \exp \left\{2 g \sum_{i=1}^{k}\left(\Phi\left(x_{i}\right)-\Phi\left(y_{i}\right)\right)\right\} \frac{\prod_{i>j}^{k}\left((c \rho)^{2}\left|x_{i}-x_{j}\right|\left|y_{i}-y_{j}\right|\right)^{2}}{\prod_{i, j}^{k}\left(c \rho\left|x_{i}-y_{j}\right|\right)^{2}}
\end{aligned}
$$


где $c \rho$ - параметр нормального упорядочения.

Чтобы перейти к бозонному описанию проблемы, мы рассмотрим следующую бозонную лагранжеву плотность:

$$
\mathcal{L}_{B}=\frac{1}{2}\left(\partial_{\mu} \varphi\right)^{2}+\frac{\alpha_{0}}{2 \beta^{2}}\left(m_{+} e^{i \beta \varphi}+m_{-} e^{-i \beta \varphi}\right)
$$

где $\beta, m_{+}(x)$ и $m_{-}(x)$ следует определить. Величина $\alpha_{0}-$ константа, которую мы вводим для упрошения сравнения нашей процедуры с предыдушими работами по локальной бозонизации $[9,10]$. При $m_{+}=m_{-}=1$ эта модель совпадает с известной моделью синус-Гордон, описывающей нейтральньй кулоновский газ. Тогда $\alpha_{0} / \beta^{2}-$ это не что иное, как соответствующий коэффициент затухания [15]. Рассмотрим теперь статистическую сумму

$$
Z_{B}=\int \mathcal{D} \varphi \exp \left\{-\int d^{2} x \mathcal{L}_{B}\right\}
$$

и разложим ее в формальный ряд по коэффишиенту затухания. Анализ, справедливый в случае $m_{+}=m_{-}=1$ для каждого члена ряда, может быть непосредственно продолжен на случай, когда эти параметры не равны друг другу и не обязательно постоянны. В результате мы получим

$$
\begin{gathered}
Z_{B}=\sum_{l=1}^{\infty} \frac{1}{(l !)^{2}}\left(\frac{\alpha}{2 \beta^{2}}\right)^{2 l} \int\left(\prod_{i=1}^{l} d^{2} x_{i} d^{2} y_{i}\right)\left(\prod_{i=1}^{l} m_{+}\left(x_{i}\right) m_{-}\left(y_{i}\right)\right) \times \\
\quad \times \frac{\prod_{i>j}^{l}\left((c \rho)^{2}\left|x_{i}-x_{j}\right|\left|y_{i}-y_{j}\right|\right)^{\frac{1}{2 \pi} \beta^{2}}}{\prod_{i, j}^{l}\left(c \rho\left|x_{i}-y_{j}\right|\right)^{\frac{1}{2 \pi} \beta^{2}}}
\end{gathered}
$$

где мы ввели перенормированную величину $\alpha$ (напомним, что в корреляционных функциях в каждом порядке теории возмушений присутствуют инфракрасные и ультрафиолетовые сингулярности).

Сравнивая этот результат с уравнением (3.11), мы видим, что оба ряда совпадают, если выполнены следуюшие тождества:

$$
\beta= \pm 2 \sqrt{\pi}, \quad \alpha=g^{\prime}
$$

и

$$
\begin{aligned}
& m_{+}\left(x_{i}\right)=\left(C_{0}\left(x_{i}\right)+C_{1}\left(x_{i}\right)\right) e^{2 g \Phi\left(x_{i}\right)}, \\
& m_{-}\left(y_{i}\right)=\left(C_{0}\left(y_{i}\right)-C_{1}\left(y_{i}\right)\right) e^{-2 g \Phi\left(y_{i}\right)} .
\end{aligned}
$$

Это наш второй результат. Мы нашли бозонное представление фермионного детерминанта (3.11), задаваемое формулой (3.13) вместе с тождествами (3.15) и (3.16). Подчеркнем, что уравнения (3.15) полностью аналогичны формулам бозонизации, полученным впервые Коулменом [10], в то время как уравнения (3.16) представляют собой новый результат, относяшийся к конкретной решаемой проблеме. 


\section{4. РЕЗУЛЬТАТЫ И ПЕРСПЕКТИВЫ}

В данной работе мы обобшили ранее предложенную НМТ, описьваюшую систему одномерных сильно коррелированных частиц, на случай, когда учитываются не только процесс рассеяния вперед, но и рассеяние назад вместе с UMKLAPP-рассеянием. Мы выписали функционал перехода вакуум-вакуум в этой модели через нетривиальный фермионный детерминант (см. уравнение (3.11)). Основной результат - это бозонное представление для этого детерминанта (уравнения (3.13), (3.15) и (3.16)). Используя эти уравнения в (3.6), мы выразили статистическую сумму системы через пять скалярных полей: $\Phi, \eta, C_{0}, C_{1}$ и $\varphi$. Предыдущий опыт с моделью рассеяния вперед [4] показывает, что физические поля (описьвающие коллективные возбуждения системы) суть $\Phi$ и $\eta$. Остальные поля - это вспомогательные переменные, которые следует отынтегрировать, для того чтобы провести последуюший анализ физики нормальных мод. Гауссовы интегралы по $C_{0}$ и $C_{1}$ могут быть легко вычислены:

$$
Z=N \int \mathcal{D} \Phi \mathcal{D} \eta \mathcal{D} \varphi e^{-S_{\mathrm{eff}}[\Phi, \eta, \varphi]},
$$

где

$$
\begin{aligned}
S_{\mathrm{eff}}[\Phi, \eta, \varphi]= & \frac{1}{2} \int d^{2} x d^{2} y\left(\partial_{\mu} \eta+\epsilon_{\mu \nu} \partial_{\nu} \Phi\right)(x) b_{(\mu)}(x, y)\left(\partial_{\mu} \eta+\epsilon_{\mu \nu} \partial_{\nu} \Phi\right)(y)+ \\
& +\int d^{2} x\left[\frac{g^{2}}{2 \pi}\left(\partial_{\mu} \Phi\right)^{2}+\frac{1}{2}\left(\partial_{\mu} \varphi\right)^{2}\right]- \\
& -\frac{1}{2}\left(\frac{g^{\prime}}{2 \pi}\right)^{2} \int d^{2} x d^{2} y\left[f_{\mu}(x) U_{(\mu)}(x, y) f_{\mu}(y)\right]
\end{aligned}
$$

и

$$
\begin{aligned}
& f_{0}(x)=\operatorname{ch}(2 g \Phi+i \sqrt{4 \pi} \varphi)(x), \\
& f_{1}(x)=\operatorname{sh}(2 g \Phi+i \sqrt{4 \pi} \varphi)(x) .
\end{aligned}
$$

Анализ действия $S_{\mathrm{eff}}[\Phi, \eta, \varphi]$ выходит за рамки настоящей статьи. Эта проблема исследуется в настояшее время методом седловой точки. Мы надеемся представить наши результаты в ближайшем будушем.

Благодарности. Эта работа была частично поддержана Национальным университетом Ла-Платы и Национальным советом научных и технических исследований CONICET (Аргентина). Ч. Наон благодарен Игорю Корепанову за приглашение принять участие во Второй международной конференции по точно решаемым моделям, состоявшейся в Челябинске, Россия, в августе 1998 г. 


\section{Список литературы}

[1] J. Voit. Rep. Progr. Phys. 1995. V. 58. P. 977.

[2] F. Haldane. J. Phys. C. 1981. V. 14. P. 2585.

[3] S. Tomonaga. Progr. Theor. Phys. 1950. V. 5. P. 544; J. Luttinger. J. Math. Phys. 1963. V. 4. P. 1154; E. Lieb, D. Mattis. J. Math. Phys. 1965. V. 6. P. 304.

[4] C. M. Naón, M. C. von Reichenbach, M. L. Trobo. Nucl. Phys. [FS] B. 1995. V. 435. P. 567; D. G. Barci, C.M. Naón. Int. J. Mod. Phys. A. 1998. V. 13. P. 1169; M. V. Manías, C. M. Naón, M. L. Trobo. Nucl. Phys. [FS] B. 1998. V. 525. P. 721.

[5] W. Thirring. Ann. Phys. 1958. V. 3. P. 91; B. E. Kopenuн. ТМФ. 1979. T. 41. C. 169

[6] B. Klaiber. The Tirring model. In: Lectures in Theoretical Physics. Proc. of Int. Conf. Held in Boulder, Colorado, USA, 1966. V. 10A. Eds A. Barut, W. Brittin. New York: Gordon and Breach, 1967. P. 141.

[7] A. Luther, V. J. Emery. Phys. Rev. Lett. 1974. V. 33. P. 589; R. Heidenreich, B. Schroer, R. Seiler, D. A. Uhlenbrock. Phys. Lett. A. 1975. V. 54. P. 119.

[8] V. J. Emery, A. Luther, I. Peschel. Phys. Rev. B. 1976. V. 13. P. 1272.

[9] C. Naón. Phys. Rev. D. 1985. V. 31. P. 2035.

[10] S. Coleman. Phys. Rev. D. 1975. V. 11. P. 2088; S. Coleman, R. Jackiw, L. Susskind. Ann. Phys. (N. Y.). 1975. V. 93. P. 267.

[11] J. Sólyom. Adv. in Phys. 1979. V. 28. P. 201.

[12] K. Fujikawa. Phys. Rev. Lett. 1979. V. 42. P. 1195; Phys. Rev. D. 1980. V. 21. P. 2848.

[13] R. Jackiw, R. Rajaraman. Phys. Rev. Lett. 1985. V. 54. P. 1219; V. 55. P. 224; D. Cabra, F. Schaposnik. J. Math. Phys. 1989. V. 30. P. 816.

[14] Kang Li, Carlos Naón. J. Phys. A: Math. Gen. 1998. V. 31. P. 7929.

[15] S. Samuel. Phys. Rev. D. 1978. V. 18. P. 1916. 